Vol. 46 (1992) [159-166]

\title{
DIRICHLET SERIES WITH POSITIVE REAL PART
}

\section{N. SAMARIS}

We consider the sequence $\Lambda=\left\{0<\lambda_{2}<\lambda_{2}<\ldots\right\}$, for which $\lambda_{n} \rightarrow+\infty$. We denote by $P D(\Lambda)$ the class of Dirichlet's series having the form $F(s)=$ $\sum_{n=0}^{\infty} a_{n} \exp \left\{-\lambda_{n} s\right\}\left(a_{0}=1\right)$ defined in the half plan Re $s>0$ converging absolutely and $\operatorname{Re} F \geqslant 0$. If $N_{0}=\{0,1,2, \ldots\}$ then the class $P D\left(N_{0}\right)$ coincides with the Caratheodory's class $P$. In this paper some classical results holding for the class $P$ are generalised in any class $P D(\Lambda)$. In special cases for the sequence $\Lambda$ extreme problems are examined in the class $P D(\Lambda)$.

\section{INTRODUCTION}

We consider the sequence $\Lambda=\left\{0=\lambda_{0}<\lambda_{1}<\lambda_{2}<\ldots\right\}$, for which $\lambda_{n} \rightarrow+\infty$. We denote by

(a) $D(\Lambda)$ the class of Dirichlet's series having the form

$$
F(s)=\sum_{n=0}^{\infty} \alpha_{n} \exp \left\{-\lambda_{n} s\right\} \quad\left(\alpha_{0}=1\right)
$$

defined in the half-plane Re $s>0$ and converging absolutely;

(b) $P D(\Lambda)$ the class

$$
\{F \in D(\Lambda): \operatorname{Re} F \geqslant 0\}
$$

(c) $D$ the union of all classes $D(\Lambda)$ and by $P D$ the union of all classes $P D(\Lambda)$.

If we set $\exp \{-\operatorname{Re} s\}=r,-\operatorname{Im} s=t(0 \leqslant r<1,-\infty<t<+\infty)$ then every $F(s) \in D$ can be written in the form

$$
\tilde{F}(r, t)=1+\sum_{n=1}^{\infty} \alpha_{n} r^{\lambda_{n}} \exp \left\{i \lambda_{n} t\right\}
$$

If $N_{0}=\{0,1,2, \ldots\}$, then the class $P D\left(N_{0}\right)$ coincides with the Caratheodory's class $P$.

Received 2 August 1991

Copyright Clearance Centre, Inc. Serial-fee code: 0004-9729/92 \$A2.00+0.00. 
In [1] the inequality $\left|\alpha_{n}\right| \leqslant 2$, which is true for the class $P$, is generalised for the class $P D$.

In [2] it is shown that if $f \in P$, then $f$ is an extreme point of the class $P$ if and only if $\left|\alpha_{1}\right|$ takes the maximal possible value, that is $\alpha_{1}=2 \exp \{i \varphi\}$, or, equivalently,

$$
f(z)=(1+\exp \{i \varphi\} z)(1-\exp \{i \varphi\} z)^{-1}
$$

In the present paper some results holding for the class $P$ are generalised in the class $P D$.

The form of extreme points of a class $D(\Lambda)$ is decisively affected by the structure of the sequence $\Lambda$, hence the solution of this problem is difficult in the general case. This assertion is also implied by Remark 2 of Theorem 2, Theorem 3 and Theorem 4 .

Remark 2 of Theorem 2 shows how to find all the extreme elements of a class $P D(\Lambda)$, if the values of the sequence $\Lambda-\{0\}$ form a linearly independent set with respect to the field of rationals.

Theorems 3 and 4 examine, in some specific cases for the sequence $\Lambda$, the form of the series

$$
\sum_{n=0}^{\infty} \alpha_{n} \exp \left\{-\lambda_{n} s\right\} \in P D(\Lambda)
$$

when $\left|\alpha_{1}\right|$ takes the maximal possible value.

The following lemma from classical Harmonic analysis will be used in the proofs of the theorems.

LEMmA 1. If $f(x)$ is an integrable function in $\mathbb{R}$,

$$
\widehat{f}(t)=\int_{\mathbb{R}} f(x) \exp \{-i t x\} d x
$$

is the Fourier transform of $f$ and $\operatorname{Re} f \geqslant 0$; then

$$
|\widehat{f}(t)+\overline{\hat{f}}(-t)| \leqslant 2 \operatorname{Re} \widehat{f}(0), \quad \text { for every } t \in \mathbb{R} .
$$

The proof is obvious.

TheOREM 2. If

$$
F(s)=\sum_{n=0}^{\infty} \alpha_{n} \exp \left\{-\lambda_{n} s\right\} \in D
$$

then the following are equivalent:

(i) $F(s) \in P D$. 
(ii) $\left|F(s)-A_{k}(s) \exp \left\{\lambda_{k} \operatorname{Re} s\right\}-\bar{A}_{k}(s) \exp \left\{-\lambda_{k} \operatorname{Re} s\right\}\right| \leqslant 2 \operatorname{Re} A_{k}(s)$ where

$$
\begin{gathered}
A_{k}(s)=\left[F_{k}(s) \exp \left\{\lambda_{k} \operatorname{Re} s\right\}-F_{k}(-\bar{s}) \exp \left\{-\lambda_{k} \operatorname{Re} s\right\}\right] \\
{\left[\exp \left\{\lambda_{k} \operatorname{Re} s\right\}-\exp \left\{-\lambda_{k} \operatorname{Re} s\right\}\right]^{-2}} \\
F_{k}(s)=\sum_{n=0}^{k} \alpha_{n} \exp \left\{-\lambda_{n} s\right\}, \quad k=1,2, \ldots
\end{gathered}
$$

(iii) Proposition (ii) is true for at least one natural number $k$.

(iv) $\operatorname{Re}\left[\sum_{n=0}^{k} \alpha_{n}\left(1-\lambda_{n} / \lambda_{k}\right) \exp \left\{i \lambda_{n} t\right\}\right] \geqslant 0, \quad k=1,2, \ldots, \quad t \in \mathbb{R}$.

Proof: (i) $\Rightarrow$ (ii). Let $\sigma>0, c>0$ and

$$
P(x)=\sum_{n=0}^{\infty} \alpha_{n} \exp \left\{-\lambda_{n} \sigma\right\}\left(\frac{\exp \left\{-i \lambda_{n} x\right\}}{c^{2}+x^{2}}\right)
$$

Since

$$
\left(\frac{1}{c^{2}+x^{2}}\right)^{\widehat{\lambda}}=\frac{\pi}{c} \exp \{-c|t|\}
$$

it follows that $\quad \widehat{P}(t)=\frac{\pi}{c} \sum_{n=0}^{\infty} \alpha_{n} \exp \left\{-\lambda_{n} \sigma-c\left|t-\lambda_{n}\right|\right\}$.

Applying Lemma 1, the function $P$, for $t \in\left[\lambda_{k}, \lambda_{k-1}\right]$ becomes

$$
\begin{gathered}
\mid F_{k}(\sigma-c) \exp \{-c t\}+F(\sigma+c) \exp \{c t\}-F_{k}(\sigma+c) \exp \{c t\} \\
+\bar{F}(\sigma+c) \exp \{-c t\} \mid \leqslant 2 \operatorname{Re} F(\sigma+c)
\end{gathered}
$$

For $t=\lambda_{k}$ and $\sigma \rightarrow 0$ it becomes

$$
\begin{gathered}
\left|F_{k}(-c) \exp \left\{-c \lambda_{k}\right\}+F(c) \exp \left\{c \lambda_{k}\right\}-F_{k}(c) \exp \left\{c \lambda_{k}\right\}+\bar{F}(c) \exp \left\{-c \lambda_{k}\right\}\right| \\
\leqslant 2 \operatorname{Re} F(c) .
\end{gathered}
$$

If we replace the absolute value with the real part we obtain the evaluation

$$
\operatorname{Re} A_{k}(c) \geqslant 0
$$

Also, taking the square of $(*)$, we obtain

$$
\left|F(c)-A_{k}(c) \exp \left\{c \lambda_{k}\right\}-\bar{A}_{k}(c) \exp \left\{-c \lambda_{k}\right\}\right|^{2} \leqslant\left[2 \operatorname{Re} A_{k}(c)\right]^{2}
$$


which, for $s=c$, is the required result.

The general case, where $s=c+i \tau(\operatorname{Re} s>0)$, is immediately obtained by substituting $F_{\tau} \in P D(\Lambda)$ for $F$ in the last inequality, where $F_{\tau}(\omega)=F(\omega+i \tau)$.

(iii) $\Rightarrow$ (i) By (iii), it is obvious that

$\operatorname{Re} F(s) \geqslant\left(\exp \left\{\lambda_{k} \operatorname{Re} s\right\}+\exp \left\{-\lambda_{k} \operatorname{Re} s\right\}-2\right) \operatorname{Re} A_{k}(s) \geqslant 0$.

(i) $\Rightarrow$ (iv) The inequality $\operatorname{Re} A_{k}(c-i t) \geqslant 0$ is equivalent to

$$
\operatorname{Re}\left\{\sum_{n=0}^{k-1} \alpha_{n} \exp \left\{i \lambda_{n} t\right\} \frac{\exp \left\{c\left(\lambda_{k}-\lambda_{n}\right)\right\}-\exp \left\{c\left(\lambda_{n}-\lambda_{k}\right)\right.}{\exp \left\{c \lambda_{k}\right\}-\exp \left\{-c \lambda_{k}\right\}}\right\} \geqslant 0
$$

which, for $c \rightarrow 0$, gives the required result.

(iv) $\Rightarrow$ (i) If

$$
f(z)=\sum_{n=0}^{k-1} \alpha_{n}\left(1-\frac{\lambda_{n}}{\lambda_{k}}\right) \exp \left\{-\lambda_{n}(1+z)(1-z)^{-1}\right\}
$$

then the function $f$ is bounded in the disc $U=\{|z|<1\}$, because

$$
\operatorname{Re}\left[(1+z)(1-z)^{-1}\right]>0, \quad \text { for every } z \in U .
$$

Furthermore, $\operatorname{Re} f(z) \geqslant 0$ almost everywhere in $\partial U=\{|z|=1\}$ because

$$
\operatorname{Re}\left[(1+z)(1-z)^{-1}\right]=0 \quad \text { almost everywhere in } \quad \partial U=\{|z|=1\} .
$$

From the Poisson integral of the function $f$, it follows that $\operatorname{Re} f(z)>0$, for every $z \in U$, or

$$
\operatorname{Re}\left\{\sum_{n=0}^{k-1} \alpha_{n}\left(1-\frac{\lambda_{n}}{\lambda_{k}}\right) \exp \left\{-\lambda_{n} s\right\}\right\}>0, \text { when Re } s>0 .
$$

For $k \rightarrow+\infty$, it follows that $F(s) \in P D$.

Remark 1. From Part (ii) of Theorem 2, the following evaluation for $|F(s)|$ follows:

$$
\begin{aligned}
& \left|A_{k}(s) \exp \left\{\lambda_{k} \operatorname{Re} s\right\}+\bar{A}_{k}(s) \exp \left\{-\lambda_{k} \operatorname{Re} s\right\}\right|-2 \operatorname{Re} A_{k}(s) \leqslant|F(s)| \\
& \quad \leqslant\left|A_{k}(s) \exp \left\{\lambda_{k} \operatorname{Re} s\right\}+\bar{A}_{k}(s) \exp \left\{-\lambda_{k} \operatorname{Re} s\right\}\right|+2 \operatorname{Re} A_{k}(s), \quad k=1,2, \ldots
\end{aligned}
$$

For $k=1$ and

we have

$$
F(r, t)=\sum_{n=0}^{\infty} \alpha_{n} r^{\lambda_{n}} \exp \left\{i \lambda_{n} t\right\} \in P D
$$

$$
\frac{1-r^{\lambda_{1}}}{1+r^{\lambda_{1}}} \leqslant|F(r, t)| \leqslant \frac{1+r^{\lambda_{1}}}{1-r^{\lambda_{1}}} .
$$

This last inequality generalises the classical evaluation

$$
(1-r)(1+r)^{-1} \leqslant|F(r, t)| \leqslant(1+r)(1-r)^{-1}
$$

when $F \in P$ in case $F \in P D$. 
REMARK 2. For $k=1$, (iv) is equivalent to the inequality $\left|\alpha_{1}\right| \leqslant \lambda_{2}\left(\lambda_{2}-\lambda_{1}\right)^{-1}$ which is stronger than $\left|\alpha_{1}\right| \leqslant 2$, in the case $\lambda_{2}>2 \lambda$.

More generally, if for the natural number $\rho$, the numbers $\lambda_{1}, \lambda_{2}, \ldots, \lambda_{\rho}$ are linearly independent with respect to the field of rational numbers, then (ii), for $k=\rho$, yields

$$
\inf _{t \in \mathbb{E}} \sum_{n=0}^{\rho} \alpha_{n}\left(1-\frac{\lambda_{n}}{\lambda_{\rho+1}}\right) \exp \left\{i \lambda_{n} t\right\}=1-\sum_{n=1}^{\rho}\left(1-\frac{\lambda_{n}}{\lambda_{\rho+1}}\right)\left|\alpha_{n}\right| \geqslant 0
$$

(see [3], p.181).

Suppose that the linear independence for the sequence $\Lambda$ is true for every natural number $\rho$ and $F \in D(\Lambda)$. The following proposition is obvious:

$$
F(s)=\sum_{n=0}^{\infty} \alpha_{n} \exp \left\{-\lambda_{n} s\right\} \in P D(\Lambda) \text { if and only if } \sum_{n=1}^{\infty}\left|\alpha_{n}\right| \leqslant 1 .
$$

If there exist two non-zero coefficients $\alpha_{\rho}=\left|\alpha_{\rho}\right| \exp \{i \vartheta\}, \alpha_{k}=\left|\alpha_{k}\right| \exp \{i \varphi\}$ and $0<\varepsilon<\min \left\{\left|\alpha_{\rho}\right|,\left|\alpha_{k}\right|\right\}$,

$$
\left|\alpha_{\rho} \pm \varepsilon \exp \{i \vartheta\}\right|+\left|\alpha_{\lambda} \mp \varepsilon \exp \{i \varphi\}\right|=\left|\alpha_{\rho}\right|+\left|\alpha_{\lambda}\right| .
$$

Consequently, $F(s)$ is an extreme element of the class $P D(\Lambda)$ if and only if it has the form

$$
F(s)=1+\alpha \exp \left\{-\lambda_{k} s\right\}
$$

where $|\boldsymbol{\alpha}|=1, k=1,2, \ldots$

THEOREM 3. If for $\Lambda=\left\{0=\lambda_{0}<\lambda_{1}<\lambda_{2}<\ldots\right\}$ it is true that

$$
\begin{gathered}
\lambda_{4}+\lambda_{1} \geqslant 2 \lambda_{2}, \quad \lambda_{k+4}-\lambda_{k-1} \geqslant 2 \lambda_{2}, \quad k=1,2, \ldots \\
F(r, t)=\sum_{n=0}^{\infty} \alpha_{n} r^{\lambda_{n}} \exp \left\{i \lambda_{n} t\right\} \in P D(\Lambda)
\end{gathered}
$$

and

then the following propositions are equivalent:

(i) $\alpha_{1}=\lambda_{2}\left(\lambda_{2}-\lambda_{1}\right)^{-1} \exp \{i \varphi\}$;

(ii) $\lambda_{k}=k \lambda_{1}, \alpha_{k}=2 \exp \{i k \varphi\}, k=1,2, \ldots$, or

$$
F(r, t)=\left[1+r^{\lambda_{1}} \exp \left\{i\left(t \lambda_{1}+\varphi\right)\right\}\right]\left[1-r^{\lambda_{1}} \exp \left\{i\left(t \lambda_{1}+\varphi\right)\right\}\right]^{-1} .
$$

THEOREM 4. If for $\Lambda=\left\{0=\lambda_{0}<\lambda_{1}<\lambda_{2}<\ldots\right\}$ it is true that $\lambda_{k}-\lambda_{k-1} \geqslant \lambda_{1}$, $k=1,2, \ldots$ and

$$
F(r, t)=\sum_{n=0}^{\infty} \alpha_{n} r^{\lambda_{n}} \exp \left\{i \lambda_{n} t\right\} \in P D(\Lambda)
$$


then the following propositions are equivalent:

(i) $\alpha_{1}=2 \exp \{i \varphi\}$;

(ii) $\lambda_{k}=k \lambda_{1}, \alpha_{k}=2 \exp \{i k \varphi\}$, or

$$
F(r, t)=\left[1+r^{\lambda_{1}} \exp \left\{i\left(t \lambda_{1}+\varphi\right)\right]\left[1-r^{\lambda_{1}} \exp \left\{i\left(t \lambda_{1}+\varphi\right)\right]^{-1} .\right.\right.
$$

Proof of TheOREM 3: If we consider the function

$$
F\left(r, t+\frac{\pi-\varphi}{\lambda_{1}}\right) \in P D(\Lambda)
$$

then the general case is reduced to $\alpha_{1}=-\lambda_{2}\left(\lambda_{2}-\lambda_{1}\right)^{-1}$. If

$$
h_{r}(x)=\frac{\sin ^{2} \delta x}{x^{2}} F(r, t), \quad P(t)=\pi\left(\frac{\sin ^{2} \delta x}{x^{2}}\right)=\sup (0,2 \delta-|t|)
$$

then $\quad \widehat{h}_{r}(t)=\sum_{n=0}^{\infty} \alpha_{n} r^{n} P\left(t-\lambda_{n}\right)$

and

$$
\lim _{r \rightarrow 0} \hat{h}_{r}(0)=0
$$

whenever $2 \delta=\lambda_{2}$. Applying Lemma 1 in the function $h_{r}$ we have that

$$
\lim _{r \rightarrow 1}\left|\widehat{h}_{r}(t)+\widehat{h}_{r}(-t)\right|=0
$$

or

$$
\sum_{n=0}^{\infty} \alpha_{n} P\left(t+\lambda_{n}\right)+\sum_{n=0}^{\infty} \bar{\alpha}_{n} P\left(t-\lambda_{n}\right)=0
$$

The set $\left\{\varepsilon: 0<\varepsilon<\lambda_{1}, \lambda_{3}-\varepsilon>\lambda_{2}\right\}$ is an interval. Setting $t=\varepsilon$ in (**), we have

$$
2 P(\varepsilon)+\alpha_{1} P\left(\varepsilon+\lambda_{1}\right)+\bar{\alpha}_{1} P\left(\lambda_{1}-\varepsilon\right)+\bar{\alpha}_{2} P\left(\lambda_{2}-\varepsilon\right)=0, \quad \text { or } \alpha_{2}=2
$$

From the inequalities $\lambda_{2} \geqslant 2 \lambda_{1}$ (since $\left|\alpha_{1}\right| \leqslant 2$ ), $\lambda_{4}+\lambda_{1} \geqslant 2 \lambda_{2}$, it follows that the set

$$
\left\{\varepsilon: \lambda_{1}>\varepsilon>0,0 \leqslant \lambda_{2}-2 \lambda_{1}+\varepsilon<\lambda_{2}, \lambda_{4}+\lambda_{1}-\lambda_{2}-\varepsilon>\lambda_{2}\right\}
$$

is an interval.

Setting $t=\lambda_{2}-\lambda_{1}+\varepsilon$ in $(* *)$ we have

$$
2 P\left(\lambda_{2}-\lambda_{1}+\varepsilon\right)+\bar{\alpha}_{1} P\left(\lambda_{2}-2 \lambda_{1}+\varepsilon\right)+\bar{\alpha}_{2} P\left(\lambda_{1}-\varepsilon\right)+\bar{\alpha}_{3} P\left(\lambda_{3}-\lambda_{2}+\lambda_{1}-\varepsilon\right)=0
$$

or

$$
-\alpha_{1} \varepsilon+2 \lambda_{1} \alpha_{1}+2 \lambda_{2}+\alpha_{3} P\left(\lambda_{3}-\lambda_{2}+\lambda_{1}-\varepsilon\right)=0 \text {. }
$$


From the last equality it follows that

$P\left(\lambda_{3}-\lambda_{2}+\lambda_{1}-\varepsilon\right) \neq 0$
or $P\left(\lambda_{3}-\lambda_{2}+\lambda_{1}-\varepsilon\right)=2 \lambda_{2}-\lambda_{3}-\lambda_{1}+\varepsilon, \quad \alpha_{3}=\alpha_{1} \quad$ and $\lambda_{3}=3 \lambda_{1}$.

In the same manner, if we set $t=\lambda_{2}+\varepsilon$ in $(* *)$, we obtain the relations $\alpha_{4}=2$ and $\lambda_{4}=2 \lambda_{2}$.

Suppose that for $n \leqslant k+3$ the equalities $\alpha_{n}=\alpha_{n-2}, \lambda_{n}=n \lambda_{1}$ when $n$ is odd and $n=(n / 2) \lambda_{2}$ when $n$ is even, hold. We will examine the case $n=k+4$, when $k$ is even.

First, the following inequalities are true:

$$
\begin{aligned}
& 0<\lambda_{k+2}-\lambda_{k+1}<\lambda_{2} \text { because } \lambda_{k+2}=\lambda_{k}+\lambda_{2} \\
& 0<\lambda_{k+3}-\lambda_{k+2}<\lambda_{2} \text { because } \lambda_{k+2}=\frac{1}{2}(k+2) \lambda_{2}, \lambda_{k+3}=(k+3) \lambda_{1}, \lambda_{2} \geqslant 2 \lambda_{1} \\
& \lambda_{2}<\lambda_{k+5}-\lambda_{k+2} \quad \text { because } \lambda_{k+5}-\lambda_{k}>2 \lambda_{2} .
\end{aligned}
$$

The above inequalities assure us that the set

$$
\left\{\varepsilon>0,0<\lambda_{k+2}-\lambda_{k+1}-\varepsilon<\lambda_{2}, 0<\lambda_{k+3}-\lambda_{k+2}-\varepsilon<\lambda_{2}<\lambda_{k+5}-\lambda_{k+2}-\varepsilon\right\}
$$

is an interval.

If we set $t=\lambda_{2}+\lambda_{k}+\varepsilon=\lambda_{k+2}+\varepsilon$ in the relation $(* *)$, then

or

$$
\begin{gathered}
\alpha_{k+1} P\left(\lambda_{k+2}-\lambda_{k+1}+\varepsilon\right)+\alpha_{k+2} P(\varepsilon)+\alpha_{k+3} P\left(\lambda_{k+3}-\lambda_{k+2}-\varepsilon\right) \\
+\alpha_{k+4} P\left(\lambda_{k+4}-\lambda_{k+2}-\varepsilon\right)=0 \\
-2 \varepsilon+\alpha_{k+4} P\left(\lambda_{k+4}-\lambda_{k+2}-\varepsilon\right)=0 .
\end{gathered}
$$

The last equality says that

or

$$
\begin{gathered}
P\left(\lambda_{k+4}-\lambda_{k+2}-\varepsilon\right) \neq 0 \\
P\left(\lambda_{k+4}-\lambda_{k+2}-\varepsilon\right)=\lambda_{2}-\lambda_{k+4}+\lambda_{k+2}+\varepsilon, \\
\alpha_{k+4}=2, \quad \lambda_{k+4}=\lambda_{k+2}+\lambda_{2}=\frac{1}{2}(k+4) \lambda_{2} .
\end{gathered}
$$

In case $k$ is odd we can prove in the same manner that $\lambda_{k+4}=(k+4) \lambda_{1}$ and $\alpha_{k+4}=\alpha_{1}$.

By the inequality

$$
k \lambda_{2}<(2 k+1) \lambda_{1}<(k+1) \lambda_{2}, \quad k=1,2, \ldots,
$$


it follows that $\lambda_{2}=2 \lambda_{1}$.

Proof of Theorem 4: If we consider the function

$$
F\left(r, \frac{t-\varphi-\pi}{\lambda_{1}}\right) \in P D(\Lambda)
$$

then Theorem 4 is reduced to the case where $\alpha_{1}=-2, \lambda_{1}=1$.

From the relation

$$
\left|\alpha_{1}\right| \leqslant \lambda_{2}\left(\lambda_{2}-\lambda_{1}\right)^{-1}
$$

it follows that $\lambda_{2}=2 \lambda_{1}=2$.

If we set $t=\lambda_{1}$ in $(* *)$ of Theorem 3 , then we have that $\alpha_{2}=2$.

Suppose that for $k=k_{0}$ it is true that $\lambda_{k}=k$ and $\alpha_{k}=2(-1)^{k}$. If we set $t=\lambda_{k}$ in $(* *)$ we have

or

$$
\alpha_{k} P(0)+\alpha_{k-1} P\left(\lambda_{k}-\lambda_{k-1}\right)+\alpha_{k+1} P\left(\lambda_{k+1}-\lambda_{k}\right)=0
$$

or

$$
\begin{aligned}
& 2(-1)^{k}+\alpha_{k+1} P\left(\lambda_{k+1}-\lambda_{k}\right)=0 \\
& \alpha_{k+1}\left[2-\left(\lambda_{k+1}-\lambda_{k}\right)\right]=2(-1)^{k} .
\end{aligned}
$$

Combining the last equality with the inequalities

$$
\begin{gathered}
\left|\alpha_{k+2}\right| \leqslant 2, \quad \lambda_{k+1}-\lambda_{k} \geqslant 1 \\
\lambda_{k+1}=\lambda_{k}+1=k \quad \text { and } \quad \alpha_{k+1}=2(-1)^{k+1} .
\end{gathered}
$$

we have

\section{REFERENCES}

[1] N. Artemiadis, 'Quelques resultat sur les transformees de Fourier avec applications', Bull. Sc. Math. 97 (1973), 177-191.

[2] F. Holland, 'The extreme points of a class of functions with positive real part', Math. Ann. 202 (1973), 85-87.

[3] Y. Katznelson, An introduction to harmonic analysis (John Wiley and Sons. Inc., New York, 1968). 